\title{
An Assessment of Comparative Marginal Costs to Non-Robotic Surgery for Radical Prostatectomy amongst Public Patients
}

\author{
Josephine Sau Fan Chow ${ }^{1,2,3 *}$, John Slater ${ }^{4}$, Les Bokey ${ }^{1,5,6}$, Pascal Mancuso ${ }^{5}$ \\ ${ }^{1}$ South Western Sydney Local Health District, Sydney, Australia \\ ${ }^{2}$ The University of Sydney, Sydney, Australia \\ ${ }^{3}$ The University of New South Wales, Sydney, Australia \\ ${ }^{4}$ South Australia Health, Adelaide, Australia \\ ${ }^{5}$ Liverpool Hospital, Sydney, Australia \\ ${ }^{6}$ Western Sydney University, Sydney, Australia \\ Email: *Josephine.Chow@health.nsw.gov.au
}

How to cite this paper: Chow, J. S. F., Slater, J., Bokey, L., \& Mancuso, P. (2021). An Assessment of Comparative Marginal Costs to Non-Robotic Surgery for Radical Prostatectomy amongst Public Patients. Journal of Service Science and Management, 14, 399-411.

https://doi.org/10.4236/jssm.2021.144025

Received: May 11, 2021

Accepted: July 27, 2021

Published: July 30, 2021

Copyright $\odot 2021$ by author(s) and Scientific Research Publishing Inc. This work is licensed under the Creative Commons Attribution International License (CC BY 4.0).

http://creativecommons.org/licenses/by/4.0/

Open Access

\begin{abstract}
Objective: Surgical procedures assisted by robotic technology have been increasing in uptake, particularly in private hospitals. Unlike most of the studies on robotic technology which were sponsored by pecuniary interests of the dominant Monopoly supplier, this paper is an independent investigators' initiated study of comparative health system costs in a Local Health District on Robotic Surgery (RS) versus Non-RS for patients undergoing prostatectomy amongst a cohort of public patients. The report was limited to understanding the cost implications of RS and productivity savings associated with it via consistently reduced length of stay (LOS). Patient benefits were not formally measured or assessed. Methods: Estimated marginal costs of both RS and Non-RS were ascertained via cost modelling Activity Based Funding (ABF) cost bucket categories for each inpatient separation for Non-RS radical prostatectomy and comparing these to actual costs for patients undergoing RS for radical prostatectomy. This approach compared the expected non-theatre costs of both RS and Non-RS, and considered appropriate cost bucket comparators for operating theatre costs. Results: The results are in congruence with established literature, RS costs are higher than Non-RS. Specifically, this study found that RS yielded a productivity saving to the health system of two days shorter LOS, than Non-RS. However, the marginal cost per separation of RS prostatectomy is $\$ 3086$ higher than Non-RS prostatectomy in the instruments and consumables costs. Any potential savings associated with reduced LOS do not offset the higher marginal costs associated with RS. Conclusion: Instruments and consumables for RS remain as monopoly products, save for
\end{abstract}


competitive forces; the health system costs for RS are likely to remain significantly higher than non-RS.

\section{Keywords}

Robotic Surgery, Prostatectomy, Cost Modelling, Activity Based Funding

\section{Introduction}

Robotic assisted surgery has increasingly popular (Yaxley et al., 2016; Wallis et al., 2018). However, as both clinical and cost effectiveness evidence of Robotic surgery remains equivocal, primarily due to research study design (lack of randomised control trial evidence) and with considerable favourable literature sponsored by pecuniary interests of a dominant Monopoly supplier (Basto et al., 2016) the development of Robotic surgery program and the associated procurement of the clinical robot faces challenges.

Prostate cancer (PC) is one of the most common cancer diagnoses in men globally and it has been reported one in five men in Australia is diagnosed with PC before the age of 85 (Ferlay et al., 2015; Bray et al., 2018; Australian Institute of Health and Welfare, 2017a, 2017b). Cancer confined to the prostate (localised prostate cancer) can be treated in several ways, most commonly being active surveillance, radical prostatectomy, radiotherapy and recently robot-assisted radical prostatectomy (RARP) (Sanda et al., 2018; Hamdy et al., 2016).

Radiotherapy has reported side effects including bowel dysfunction, while urinary incontinence and erectile dysfunction are more common after radical prostatectomy (Donovan et al., 2016). RARP has become increasingly popular due to minimising side effects, but there is currently limited evidence of better oncologic or functional outcomes compared to open prostatectomy or other treatments (Yaxley et al., 2016; Wallis et al., 2018). In addition, RARP cancer surgery has been reported a $71 \%$ reduction in patient length of stay (LOS) (Basto et al., 2016).

Most of the published literatures focus on choice between open radical prostatectomy, radiotherapy and active surveillance, with limited publications specifically focused on RARP (Smith et al., 2019; Alemzadeh et al., 2014; Dubeck, 2014; Bolenz et al., 2014; Tandogdu et al., 2015). RARP is increasingly being offered to patients for free in some public hospitals in Australia. Patient's choice however, requires patients' preferences to be well informed. The Prostatectomy versus Radiotherapy for Early-stage PRostatE Cancer (PREPaRE) study (Smith et al., 2019) was conducted by the investigator team to understand how to best support men diagnosed with PC in choosing the treatment that best suits their values/preferences, when robotic prostatectomy and radiotherapy are equally appropriate to offer them, in that they offer equivalent cure rates. The study concluded that treatment choice is largely dependent on clinicians' (mainly urologists') recommendations. Participants mistakenly believed that RARP pro- 
vides a more definitive cure for PC than radiotherapy suggesting incomplete/biased information provision and/or a need to more carefully check understanding.

It is crucial to monitor patient safety when introducing a new clinical procedure such as robotic surgery. Alemzadeh et al. (Alemzadeh et al., 2014) reported 5374 adverse robotic events as a result of systems malfunction or inadequate technical competence, and of which 86 resulted in death and 455 with serious injuries. Furthermore, in a review by Dubeck (2014) there were 722 safety events associated with robotic procedures, of which 177 (24.5\%) were reported as causing harm to patients resulting in 10 fatalities.

Productivity is the relationship between inputs and outputs. If any economic activity can do more (greater outputs), with the same or less resources, a productivity gain has occurred. Limited literature has documented reductions in LOS associated with some types of robotic surgery. Others based on systematic reviews have found the opposite result (Bolenz et al., 2014; Tandogdu et al., 2015). If the limited evidence is correct, reductions in LOS could translate to lower costs for the health system for these patients associated with ward and nursing costs. However, LOS productivity gains in the health system are rarely realised as a saving whereby the hospital beds freed up faster for the next patient to occupy can translate to higher levels of throughput and higher costs.

In Australia, all surgery will attract variable Activity Based Funding (ABF) revenue. Under ABF pricing, each episode of care is completely dependent on the Diagnosis Related Group (DRG) of the patient and individual patient characteristics such as LOS, and a range of other specific patient characteristics. The literature has accurately documented, albeit for prostatectomies, that ABF does not adequately cover the costs of robotic surgery (Basto et al., 2016). Moreover, the fact that a surgery uses a robot will not attract a higher case payment via recoding the patient to a higher paying DRG (Markian, 2014). Current cost modelling for inpatient activity is retrospective and top down whilst some actual costs are recorded; other costs attributed to the patient's hospital stay are apportioned across cost buckets using averages. In conjunction with the commencement of RS at a major teaching hospital in Australia in 2017, a research protocol was developed to support the collection of cost modelling data for robotic prostatectomy. This major teaching hospital provides a health service of international standing, with 23 operating theatres, capacity for 877 beds, diagnostic and imaging services, emergency and trauma care, maternity, paediatric, cancer care, mental health, ambulatory care, allied health and medical and surgical services from birth to aged care.

\section{Methods}

The study aims to facilitate cost modelling to identify the cost and relative productivity of robotic and radical prostatectomy surgery. This will support analysis with respect to cost and $\mathrm{ABF}$ revenues, guiding decision making to in- 
form the model of care for public robotic prostatectomy and financial sustainability to the hospital. The underlying hypothesis to be tested is whether or not robotic prostatectomy productivity gains associated with improved LOS can offset the high costs of consumable instruments used in robotic surgery.

The specific aims supporting the hypothesis of this study is twofold being:

1) Assess differences between costs of RS and Non-RS for radical prostatectomy; and

2) Consider if productivity gains achieved by RS radical prostatectomy via lower LOS compared to Non-RS offset the known higher cost of RS consumables and RS instruments.

The data of the study is limited to RS for prostatectomy and for initial patient load in the calendar year 2017 at the study hospital. Comparative costs are limited to Non-RS prostatectomy within the Local Health District. The methodology employed in the study utilises data from the NSW Activity Based Management $(\mathrm{ABM})$ portal to:

- inform baseline marginal costs of Non-RS prostatectomy inpatient separations; and

- inform RS prostatectomy inpatient separation marginal costs that occur outside of the operating theatre and are dependent upon LOS.

Data collected for each RS prostatectomy is used to inform the marginal cost incurred for RS and includes an itemised count for each patient of consumables and instruments. Data from Surginet (local surgical database) for these patients, informs the surgical, medical, and nursing costs associated with within the operating. For Non-RS, data was extracted for three years 2014-15; 2015-16; 2016-17 for separations that had a DRG M01A/B, and where the principal procedure was Radical Prostatectomy by either Laparoscopic/open methods. Seventy-two (72) separations were identified meeting these criteria in the study Local Health District, with the majority (50) occurring at the study Hospital and 22 at the other two hospitals.

From the list of cost buckets above, the OR cost bucket is a key cost of differentiation between RS and Non-RS. By definition this includes in the ABM portal all consumables and instruments used, and importantly includes all salaries and wages, Visiting Medical Officer payments that occur in the operating theatre. These costs have been collected for each RS prostatectomy at the study Hospital in 2017, with the corresponding Surginet data informing human resource costs via describing the mix of staff and the duration in minutes of the procedure.

Per-diem estimates for marginal costs per separation for expenses that are reflected in the patient's LOS were not collected for RS patients. The costs of doing so were deemed to be excessive and unnecessary as the expected ward and medical care costs for patients post-surgery on a daily basis are expected to be identical for RS and Non-RS patients. In this regard 3 years of pooled data from the NSW ABM Portal was utilised that identifies individual costs buckets for Nurs- 
ing, Medical, Ward, and Non-clinical costs (reflecting costs that are sensitive to LOS). The defining factor for differentiating these costs at the margin between RS patients and the Non-RS patients is the LOS of the patients. For both surgical groups the actual LOS of the patient's separations was used. SPSS statistical software (Version 20) was used to assess the distribution of this sample data.

The costs buckets Allied, Critical Care, Imaging, Pathology, Pharmacy, Prosthesis, On Cost, are excluded whilst contributing to marginal costs, are assumed for radical prostatectomy to be statistically identical costs per separation, regardless of the modality of surgery. These costs are assumed to be unlikely to be influenced by LOS variations. Given this and the goal of the project to consider marginal costs differences with respect to potential productivity offsets by reduced LOS, these costs are excluded from this analysis.

For RS, cost data of consumables and instruments for 22 patients undergoing RS prostatectomy were collected in 2017 using an Excel template. Surginet data for these patients was also accessed, which showed the duration of the operation in minutes and the attending, surgical, medical, and nursing staff that was in the operating theatre. Together, staffing costs and consumables and instruments, constitute a comparator to the DRG cost bucket observed in the ABM Portal "OR". Staffing estimates were calculated using NSW Health "Remuneration Rates for VMOs" Information Bulletin (IB2017_029) which is current from 11 September 2017 through to 11 September 2018. Rates are shown for VMO Surgeons and Anaesthetists. Nursing staff costs were estimated using Registered Nursing Awards. Rates per hour were calculated per minute of the surgery duration.

South Western Sydney Local Health District Human Research Ethics Committee granted Ethics approval for this study as a Low Negligible Risk (LNR) to proceed in 2017.

\section{Results}

De-identified data was collated on costs associated with the 22 patients undergoing RS for prostatectomy during 2017 and comparative data for Non-RS for prostatectomy was sourced from the ABM portal for three years 2014-2017 involving 72 patients across the Local Health District.

The ABM portal data shows the cost breakdown for each encounter (inpatient separation) across common DRG components (cost buckets) which provide a cost breakdown for each separation. The ABM portal data for Nursing, Medical, and Ward costs for the three years 2014-15, 2015-16, and 2016-17 was assessed on a per diem basis and per separation for all Local Health District patients having Radical Prostatectomy (Table 1).

In Table 2, a key statistic is the "Per Diem Non RS". These represent the marginal costs in the sample of non-RS prostatectomy patients that are influenced by LOS. These costs are outside the operating theatre and assumed to be typical of the marginal costs incurred daily by both non-RS and RS patients. The means shown in Table 2 for these variables reflect this calculation. 
Table 1. Marginal cost elements that are sensitive to length of stay and different surgical modalities.

\begin{tabular}{|c|c|c|}
\hline Marginal Cost elements & Robotic Surgery & Non-Robotic Surgery \\
\hline \multirow{6}{*}{ OR Costs } & \multicolumn{2}{|l|}{ Itemised costs for each patient } \\
\hline & \multirow{4}{*}{$\begin{array}{l}\text { - all consumable costs associated with the surgery; } \\
\text { - robotic surgery instrument costs; } \\
\text { - operating theatre staffing costs (Surginet based on staffing } \\
\text { compliment for each patient/staff awards/VMO costs and } \\
\text { time in theatre) }\end{array}$} & OR cost bucket in the $\mathrm{ABM}^{\dagger}$ portal. \\
\hline & & This includes average cost of all Goods \& Services \\
\hline & & (excluding Prosthesis), Salary and Wages and VMO \\
\hline & & Payments for Operating Theatre cost centres. \\
\hline & \multicolumn{2}{|c|}{$\begin{array}{l}\text { Per diem nursing, medical, ward costs, non-clinical costs (statistical estimate of the median per diem costs from } 3 \\
\text { years pool data from the } \mathrm{ABM}^{\dagger} \text { portal). These costs outside the OR are considered to be identical on a per diem basis. } \\
\text { LOS }^{\ddagger} \text { differences for each separation will be the key driver of difference in marginal costs between } \mathrm{RS}^{\S} \text { and Non-RS`. }\end{array}$} \\
\hline Nursing Costs & \multicolumn{2}{|c|}{ Average cost of Nursing Salary and Wages in Clinical Service or Ward cost centres ( $\mathrm{ABM}^{\dagger}$ Portal) } \\
\hline Medical Costs & \multicolumn{2}{|c|}{ Average cost of all Medical Salary and Wages and VMO Payments in Clinical Service or Ward cost centres } \\
\hline Ward Costs & \multicolumn{2}{|c|}{$\begin{array}{l}\text { Average cost of all Goods \& Services (excluding Pathology, Imaging, Pharmacy and Prosthesis) for Clinical Service or } \\
\text { Ward cost centres }\end{array}$} \\
\hline Non Clinical & \multicolumn{2}{|c|}{ Average amount of non-clinical costs including hotel and administrative costs, non-clinical salaries and wages } \\
\hline
\end{tabular}

Note: NB. ABM Portal DRG costs buckets for Nursing, Medical, Ward, and Non-clinical are likely to be costs that are common and similar to both non-RS and RS, and will be influenced in the marginal cost calculations per separation by LOS. ${ }^{\dagger}$ Activity Based Management, ${ }^{\sharp}$ length of stay, ${ }^{\S}$ Robotic Surgery, Non-Robotic Surgery.

Table 2. Non-robotic surgery activity based management costs radical prostatectomy.

Non $\mathrm{RS}^{\dagger}$ costs: $\mathrm{ABM}^{\ddagger}$ Portal 2014-2017

\begin{tabular}{|c|c|c|c|c|c|c|c|c|c|}
\hline & & \multicolumn{5}{|c|}{ Costs likely to be influenced by $\operatorname{LOS}^{\S}$} & \multirow[b]{2}{*}{$\operatorname{LOS}(L)$} & \multirow[b]{2}{*}{ OR Costs $(0)$} & \multirow[b]{2}{*}{$\begin{array}{l}\text { All other cost } \\
\text { buckets (a) }\end{array}$} \\
\hline & & $\begin{array}{l}\text { Nursing } \\
\text { Costs per } \\
\text { diem }(x)\end{array}$ & $\begin{array}{c}\text { Medical } \\
\text { Costs per } \\
\text { diem }(y)\end{array}$ & $\begin{array}{l}\text { Ward Costs } \\
\text { per diem }(z)\end{array}$ & $\begin{array}{c}\text { Non Clinical } \\
\text { Costs per diem } \\
(t)\end{array}$ & $\begin{array}{c}\text { Per Diem non } \\
\mathrm{RS}^{\dagger} \text { Marginal } \\
\text { Costs }(q)\end{array}$ & & & \\
\hline \multirow[t]{2}{*}{ Number } & Valid & 72 & 72 & 72 & 72 & 72 & 72 & 72 & 72 \\
\hline & Missing & 0 & 0 & 0 & 0 & 0 & 0 & 0 & 0 \\
\hline Mean & & 468.992 & 140.839 & 226.844 & 204.714 & 1041.389 & 5.027 & 5840.431 & 2247.189 \\
\hline Median & & 517.217 & 146.583 & 231.857 & 217.940 & 1130.933 & 4.125 & 5830.350 & 1843.650 \\
\hline $\begin{array}{l}\text { Standard } \\
\text { Deviation }\end{array}$ & & 179.717 & 82.615 & 111.994 & 86.765 & 410.558 & 2.765 & 2596.904 & 1411.383 \\
\hline Range & & 778.24 & 355.80 & 373.59 & 380.72 & 1798.24 & 14.00 & 12395.93 & 8058.54 \\
\hline
\end{tabular}

${ }^{\dagger}$ Non-Robotic Surgery, ${ }^{\ddagger}$ Activity Based Management, 'length of stay.

As LOS is an important parameter for multiplying potential productivity savings to offset costs of RS, and for calculations of marginal costs per separation, the LOS of all patient encounters from the ABM data was statistically assessed. Bootstrapping to replicate resampling of the data 1000 times developed 95\% CI for the mean and median LOS observed in the ABM data (Table 3).

It is recommended for comparison purposes that medians are used for the assessment of marginal costs of non-RS and RS. Using the data in Table 2, this would yield an estimated marginal cost of non-RS being $\$ 12,339.10$ per separation. It is recommended that the upper limit of the $95 \%$ CI for the median LOS (5.2381 days) generated through bootstrapping is used for sensitivity analysis. 
Table 3. Length of stay for non-robotic surgery prostatectomy from the activity based management portal.

\begin{tabular}{|c|c|c|c|c|c|c|}
\hline \multicolumn{7}{|c|}{ LOS $^{\dagger}$ Non $\mathrm{RS}^{\ddagger}$ costs: $\mathrm{ABM}^{\S}$ Portal 2014-2017 } \\
\hline & & \multirow[b]{3}{*}{ Statistic } & \multicolumn{4}{|c|}{ Bootstrap } \\
\hline & & & & & \multicolumn{2}{|c|}{ BCa 95\% Confidence } \\
\hline & & & Bias & Standard Error & Lower & Upper \\
\hline \multirow[t]{2}{*}{ Number } & Valid & 72 & 0 & 0 & & \\
\hline & Missing & 0 & 0 & 0 & & \\
\hline Mean & & 5.0270 & 0.0207 & 0.3377 & 4.3479 & 5.7990 \\
\hline Median & & 4.1250 & 0.3008 & 0.4592 & 4.0000 & 5.2381 \\
\hline Standard Deviation & & 2.76506 & -0.03511 & 0.42793 & 1.92266 & 3.50518 \\
\hline Variance & & 7.646 & -0.010 & 2.328 & 3.553 & 12.371 \\
\hline Range & & 14.00 & & & & \\
\hline
\end{tabular}

${ }^{\dagger}$ Length of stay, ${ }^{\ddagger}$ Non-Robotic Surgery, ${ }^{\circledR}$ Activity Based Management, 'Unless otherwise noted, bootstrap results are based on 1000 bootstrap samples.

Table 4 provides $95 \%$ confidence intervals for the median per diem costs (\$1130.93 shown in Table 2) using bootstrapping to simulate 1000 samples of the data. This shows that per-diem cost estimates contributing to marginal cost of non RS had a median upper and lower 95\% CI of $\$ 1040$ to $\$ 1174$. The median cost of $\$ 1130$ will be used to inform marginal costs associated with LOS savings for RS. The bootstrapped upper $95 \%$ CI of $\$ 1174$ will be used in sensitivity analysis.

The data in Table 5 shows descriptive statistics of the RS data collected on OR costs and LOS. This data was bootstrapped to simulate resampling 1000 times and to develop 95\% confidence intervals for the median. Using the median costs and LOS from Table 5, and the previous median estimate of per deim costs associated with, Nursing, Wards, Medical, and Non-clinical costs from the ABM portal. The median bed days for Non-RS prostatectomy in the Local Health District are 4.125 days. The median bed days for RS prostatectomy at the study Hospital were 2.000 days, a saving of 2.125 bed days over the Non-RS modality.

The data analysis has estimated the cost comparison between RS and Non-RS for prostatectomy is as follows:

- RS prostatectomy $\$ 15,425$ per separation

- Non RS prostatectomy $\$ 12,339.10$ per separation

\section{Discussion}

This study aims to facilitate cost modelling to identify the cost and relative productivity of robotic and radical prostatectomy surgery so as to support analysis with respect to cost and $\mathrm{ABF}$ revenues, guiding decision making to inform the model of care for public robotic prostatectomy and financial sustainability to the hospital. Between the cohorts the length of stay saving associated with RS is 2 days. 
Table 4. Per diem marginal costs from the activity based management portal for non-robotic surgery.

\begin{tabular}{|c|c|c|c|c|c|c|}
\hline \multicolumn{7}{|c|}{ Per Diem Marginal costs: $\mathrm{ABM}^{\dagger}$ Portal 2014-2017 } \\
\hline & & \multicolumn{5}{|c|}{ Bootstrap $^{\dagger}$} \\
\hline & & \multirow[b]{2}{*}{ Statistic } & \multirow[b]{2}{*}{ Bias } & \multirow[b]{2}{*}{ Std. Error } & \multicolumn{2}{|c|}{ BCa 95\% Confidence } \\
\hline & & & & & Lower & Upper \\
\hline \multirow[t]{2}{*}{ Number } & Valid & 72 & 0 & 0 & & \\
\hline & Missing & 0 & 0 & 0 & & \\
\hline Mean & & 1041.3895 & -0.7140 & 47.3812 & 938.3961 & 1137.0687 \\
\hline Median & & 1130.9333 & -0.7873 & 39.8802 & 1040.7667 & 1173.9000 \\
\hline Standard Deviation & & 410.55793 & -4.01055 & 31.95074 & 351.39624 & 461.23008 \\
\hline Skewness & & -0.374 & -0.014 & 0.220 & -0.845 & -0.002 \\
\hline Std. Error of Skewness & & 0.283 & & & & \\
\hline Kurtosis & & -0.093 & 0.021 & 0.470 & -0.795 & 0.948 \\
\hline Std. error of Kurtosis & & 0.559 & & & & \\
\hline
\end{tabular}

${ }^{\dagger}$ Activity Based Management, ${ }^{*}$ Unless otherwise noted, bootstrap results are based on 1000 bootstrap samples.

Table 5. Descriptive statistics of the robotic surgery data collected on costs and length of stay.

\begin{tabular}{|c|c|c|c|c|c|c|c|}
\hline & & & \multicolumn{5}{|c|}{ Bootstrap $\dagger$} \\
\hline & & & \multirow{2}{*}{ Statistic } & \multirow{2}{*}{ Bias } & \multirow{2}{*}{ Std. Error } & \multicolumn{2}{|c|}{ Interval } \\
\hline & & & & & & Lower & Upper \\
\hline \multirow[t]{8}{*}{ Number } & Valid & $\mathrm{RS}^{\ddagger}$ Instruments & 22 & 0 & 0 & & \\
\hline & & RS_consumables & 22 & 0 & 0 & & \\
\hline & & RS_OR_Staff & 22 & 0 & 0 & & \\
\hline & & $\operatorname{LOS}^{\mathfrak{S}}$ & 22 & 0 & 0 & & \\
\hline & Missing & RS_Instruments & 0 & 0 & 0 & & \\
\hline & & RS_consumables & 0 & 0 & 0 & & \\
\hline & & RS_OR_Staff & 0 & 0 & 0 & & \\
\hline & & LOS & 0 & 0 & 0 & & \\
\hline \multirow[t]{4}{*}{ Mean } & & RS_Instruments & 2976.5786 & 0.9212 & 28.1529 & 2942.3400 & 3029.0059 \\
\hline & & RS_consumables & 2349.7845 & 4.7214 & 75.4652 & 2168.2788 & 2528.4413 \\
\hline & & RS_OR_Staff & 6255.8651 & -6.6721 & 337.9965 & 5678.7937 & 6923.5622 \\
\hline & & LOS & 2.1818 & 0.0049 & 0.2378 & 1.9091 & 2.5909 \\
\hline \multirow[t]{4}{*}{ Median } & & RS_Instruments & 2942.3400 & 0.0000 & 0.0000 & & \\
\hline & & RS_consumables & 2321.9750 & 27.7466 & 72.5804 & 2122.1588 & 2630.6200 \\
\hline & & RS_OR_Staff & 6055.4250 & -69.2225 & 260.1140 & 5656.4250 & 6320.8642 \\
\hline & & LOS & 2.0000 & 0.0000 & 0.0000 & & \\
\hline \multirow[t]{2}{*}{ Mode } & & RS_Instruments & 2942.34 & & & & \\
\hline & & RS_consumables & 2314.00 & & & & \\
\hline
\end{tabular}




\begin{tabular}{|c|c|c|c|c|c|c|}
\hline & RS_OR_Staff & 5630.40 S & & & & \\
\hline & LOS & 2.00 & & & & \\
\hline \multirow{4}{*}{$\begin{array}{l}\text { Standard } \\
\text { Deviation }\end{array}$} & RS_Instruments & 136.6237 & -21.92154 & 72.98934 & 0.00000 & 184.00737 \\
\hline & RS_consumables & 359.6822 & -10.08797 & 39.49973 & 283.92770 & 405.93624 \\
\hline & RS_OR_Staff & 1692.3855 & -86.06176 & 405.90882 & 994.03333 & 2209.11684 \\
\hline & LOS & 1.1396 & -0.14354 & 0.52091 & 0.29424 & 1.78154 \\
\hline \multirow[t]{4}{*}{ Range } & RS_Instruments & 635.55 & & & & \\
\hline & RS_consumables & 1178.44 & & & & \\
\hline & RS_OR_Staff & 7626.24 & & & & \\
\hline & LOS & 6.00 & & & & \\
\hline
\end{tabular}

†Unless otherwise noted, bootstrap results are based on 1000 bootstrap samples, ${ }^{\ddagger}$ Robotic Surgery, ${ }^{\mathfrak{S}}$ length of stay, 9 Multiple modes exist. The smallest value is shown.

The key driver of the higher marginal costs of RS, $\$ 3086$, is the instruments and consumables costs. This finding is consistent with the literature regarding RS. Whilst the supply of instruments and consumables for RS remain as monopoly products, save for competitive forces, the health system costs for RS are likely to remain significantly higher than non RS, irrespective of any productivity savings associated with reduced length of stay due to RS.

The implication of this is that any saving of bed days that RS has over Non-RS for prostatectomy is not offset by the higher OR costs associated with RS consumables and instruments. However, it is observable through the collected Surginet data, that there are multiple personnel involved in the surgery. Should staff be involved in training the cost of these staff could be removed from the cost calculations or used as a charge-back arrangement to the training surgeon and a revenue source. Another key variable that may be influenced to reduce RS costs, is that the duration of the surgery and its associated staff costs may well be lower once more surgeons are trained and become skilled with surgical robot. The times observed in the sample data may currently reflect teaching and supervision, so these times may be currently reflecting an abnormally longer duration for surgery and associated staff costs.

There are two tranches of data capture in this project, being for RS and Non-robotic surgery. The data collection and analysis in both tranches supports the assessment of marginal costs. In economics marginal costs reflect the marginal cost of production, being the change in total cost that comes from making or producing one additional item. Marginal cost assessments are useful for assessing where scale economies occur through volume, and are also useful in the assessment of technical and allocative efficiency. The method of comparison developed marginal costs of both RS and Non-RS. This utilised known methods for ascertaining cost modelling via categories or cost buckets used in $\mathrm{ABF} /$ casemix modelling, and comparing like with like regarding the expected non-theatre 
costs of both RS and Non-RS, and considering appropriate cost bucket comparators for operating theatre costs. Doing so takes full advantage of making transparent any productivity saving associated with reduced length of stay.

ABF per DRG is dependent on a specific inlier range of LOS. Should the productivity gains of reduced LOS drop below the lower trim point of the DRG, which could be possibly, be the case for prostatectomies with a $71 \%$ reduction, revenues paid under an $\mathrm{ABF}$ model could fall. This fact was well illustrated in the study by Basto et al. (2016) where LOS reductions for Robotic prostatectomies fell below the inlier trim points required for full DRG payment of the two main prostatectomy DRG categories.

It is important to note that even if the Robotic surgery resulted in patient LOS that satisfied full case DRG payment under ABF, the full casemix payment may not adequately cover the increased cost burden of the Robotic surgery. This fact was also demonstrated by Basto et al. (2016) in the context of RARP.

"This study also showed that the case-mix funding model failed to adequately reimburse public hospitals for RARP despite much more efficient use of hospital resources in terms of hospital stay and reductions in costly readmissions".

It is imperative that the potential pool of Robotic surgery patients are identified by DRG along with the DRGs of the surgical list that will be cancelled to make way for the surgery. The following is required:

- Type of surgery that will be conducted by DRG and volume

- Patient clinical characteristics (if possible the ICD10 codes) that make them suitable for alternative Robotic surgery

- Public and private patient volumes

\subsection{Limitation}

The report was limited to understanding the cost implications of RS, a known higher cost procedure, and the productivity savings associated with it via consistently reduced length of stay. Patient benefits were not formally measured or assessed in this study.

The considerable difference between RS and Non-RS for prostatectomy, $\$ 3086$ reflects the RS instrument and consumables costs. Further analysis was conducted, however, the analysis provided herein is nonetheless stable between RS and Non-RS, and RS would likely still cost more irrespective of the savings in reduced LOS.

It is important to emphasise, that this project encompasses a comparative marginal costs and technical efficiency assessment of the outputs of RS for prostatectomy versus that of Non-robotic prostatectomy. In this regard the study is not a comprehensive economic evaluation. The outcomes of prostatectomy, (the clinical benefits), whether by robotic or Non-robotic surgical approaches are clearly of the upmost importance to the District's care of its patients. The marginal benefits in terms of clinical outcomes are a much more significant study and worthy of the Local Health District's consideration at a later date in the form of a comprehensive economic evaluation. 


\subsection{Future Research}

Whilst cost comparisons are of use to inform health system costs, the true value of RS from a public hospital modality would require a more detailed cost effectiveness analysis. Such a study would be more detailed undertaking involving measuring patient outcomes in RS versus Non-RS. However, the results of this study are completely congruent with the broader literature that robotic surgical costs are higher than Non-RS costs. When patient outcomes are included in costs effectiveness studies the literature finds that these higher cost are often the determining factor for cost effectiveness of the RS to remain in doubt.

It could be argued on this basis, that Robotic surgery is a questionable investment, but ultimately the decision to proceed and the risk that entails, could be considered as an opportunity to contribute to a more robust level of evidence of clinical effectiveness via the establishment of a randomised control trial, and the establishment of a formal postgraduate qualification to ensure surgeons are credentialed to the highest standards.

With new suppliers of robotic surgical equipment entering the market, aspects of cost effectiveness may improve over time via reductions in the capital and recurrent costs of Robotic Surgery. Establishing a research agenda, free from pecuniary bias, could assist in further understanding the patient benefits of this technology.

\section{Conclusion}

Robotic surgery does represent a current substantial financial risk to public health finances, with the procurement costs of million dollars for the clinical robot, and running costs of millions per annum associated with surgery representing an opportunity cost to competing for strategic priorities, that may have a more immediate and obvious benefit to patients. As such, this paper identifies issues that should consider in developing a comprehensive plan to progress the development of robotic surgery to ameliorate risks and to contribute to a greater understanding of the research agenda. The issues herein are necessary real world operational factors that must be addressed before the project commences.

\section{Funding}

This research received no specific funding.

\section{Conflicts of Interest}

There is no conflict of interest. The authors have no financial or professional relationships which may pose a competing interest.

\section{References}

Alemzadeh, H. I. R., Raman, J., Leveson, N., \& Ravishankar, K. (2014). Safety Implications of Robotic Surgery: Analysis of Recalls and Adverse Event Reports of da Vinci Surgical Systems. The Society of Thoracic Surgeons Annual Meeting, Orlando, Florida, 
25-29 January 2014, 68-69.

Australian Institute of Health and Welfare (2017a). Australian Cancer Incidence and Mortality (ACIM) Books: Prostate Cancer. Australian Institute of Health and Welfare.

Australian Institute of Health and Welfare (2017b). Cancer in Australia 2017. Cancer Series No. 101. Cat. No. CAN 100. AIHW.

Basto, M., Sathianathen, N., Te Marvelde, L., Ryan, S., Goad, J., Lawrentschuk, N. et al. (2016). Patterns-of-Care and Health Economic Analysis of Robot-Assisted Radical Prostatectomy in the Australian Public Health System. BJU International, 117, 930-939. https://doi.org/10.1111/bju.13317

Bolenz, C., Freedland, S. J., Hollenbeck, B. K., Lotan, Y., Lowrance, W. T., Nelson, J. B. et al. (2014). Costs of Radical Prostatectomy for Prostate Cancer: A Systematic Review. European Urology, 65, 316-324. https://doi.org/10.1016/j.eururo.2012.08.059

Bray, F., Ferlay, J., Soerjomataram, I., Siegel, R. L., Torre, L. A., \& Jemal, A. (2018). Global Cancer Statistics 2018: GLOBOCAN Estimates of Incidence and Mortality Worldwide for 36 Cancers in 185 Countries. CA: A Cancer Journal for Clinicians, 68, 394-424. https://doi.org/10.3322/caac.21492

Donovan, J. L., Hamdy, F. C., Lane, J. A., Mason, M., Metcalfe, C., Walsh, E. et al. (2016). Patient-Reported Outcomes after Monitoring, Surgery, or Radiotherapy for Prostate Cancer. The New England Journal of Medicine, 375, 1425-1437. https://doi.org/10.1056/NEJMoa1606221

Dubeck, D. (2014). Robotic-Assisted Surgery: Focus on Training and Credentialing. Pennsylvania Patient Safety Authority, 11, 93-101.

Ferlay, J., Soerjomataram, I., Dikshit, R., Eser, S., Mathers, C., Rebelo, M. et al. (2015). Cancer Incidence and Mortality Worldwide: Sources, Methods and Major Patterns in GLOBOCAN 2012. International Journal of Cancer, 136, E359. https://doi.org/10.1002/ijc.29210

Hamdy, F. C., Donovan, J. L., Lane, J. A., Mason, M., Metcalfe, C., Holding, P. et al. (2016). 10-Year Outcomes after Monitoring, Surgery, or Radiotherapy for Localized Prostate Cancer. The New England Journal of Medicine, 375, 1415-1424. https://doi.org/10.1056/NEJMoa1606220

Markian, H. (2014). Robotic Surgery May Raise Costs Medicare Reimbursements Increase. The Bulletin.

https://www.bendbulletin.com/lifestyle/health/robotic-surgery-may-raise-costs/article ed44013e-5a30-5a26-84cd-eee503908cec.html

Sanda, M. G., Cadeddu, J. A., Kirkby, E., Chen, R. C., Crispino, T., Fontanarosa, J. et al. (2018). Clinically Localized Prostate Cancer: AUA/ASTRO/SUO Guideline. Part I: Risk Stratification, Shared Decision Making, and Care Options. Journal of Urology, 199, 683-690. https://doi.org/10.1016/j.juro.2017.11.095

Smith, A. B., Rincones, O., Sidhom, M., Mancuso, P., Wong, K., Berry, M. et al. (2019). Robot or Radiation? A Qualitative Study of the Decision Support Needs of Men with Localised Prostate Cancer Choosing between Robotic Prostatectomy and Radiotherapy Treatment. Patient Education and Counseling, 102, 1364-1372. https://doi.org/10.1016/j.pec.2019.02.017

Tandogdu, Z., Vale, L., Fraser, C., \& Ramsay, C. (2015). A Systematic Review of Economic Evaluations of the Use of Robotic Assisted Laparoscopy in Surgery Compared with Open or Laparoscopic Surgery. Applied Health Economics Health Policy, 13, 457-467. https://doi.org/10.1007/s40258-015-0185-2

Wallis, C. J. D., Glaser, A., Hu, J. C., Huland, H., Lawrentschuk, N., Moon, D. et al. (2018). Survival and Complications Following Surgery and Radiation for Localized 
Prostate Cancer: An International Collaborative Review. European Urology, 73, 11-20. https://doi.org/10.1016/j.eururo.2017.05.055

Yaxley, J. W., Coughlin, G. D., Chambers, S. K., Occhipinti, S., Samaratunga, H., Zajdlewicz, L. et al. (2016). Robot-Assisted Laparoscopic Prostatectomy versus Open Radical Retropubic Prostatectomy: Early Outcomes from a Randomised Controlled Phase 3 Study. The Lancet, 388, 1057-1066. https://doi.org/10.1016/S0140-6736(16)30592-X 